\title{
Análise da utilização do Questionário de Tolerância de Fagerström (QTF) como instrumento de medida da dependência nicotínica*
}

\author{
Luis SuÁrez Halty ${ }^{1}$, Maura Dumont HütTneR ${ }^{1}$, IsABEl CRistina de Oliveira NetTo ${ }^{2}$, \\ VALÉRIA A. DOS SANTOS ${ }^{3}$, GRASIELE MARTINS ${ }^{4}$
}

\begin{abstract}
Introdução: O Hospital Universitário é referência na cidade de Rio Grande, no Rio Grande do Sul, para pneumopatas crônicos, sendo importante a existência de um programa para cessação do fumo. Objetivos: Analisar a utilização do Questionário de Tolerância de Fagerström como instrumento de medida da magnitude da dependência nicotínica do paciente tabagista e obter subsídios para planejar a conduta terapêutica mais adequada. Material e método: Aplicação do Questionário de Tolerância de Fagerström em pacientes adultos fumantes regulares, dos setores de Clínica Médica e Pneumologia do Hospital Universitário e Santa Casa de Rio Grande durante o período de 12 meses. Foram preenchidos 301 questionários válidos, sendo 40,5\% dos entrevistados do sexo feminino e 59,5\% do masculino. A média de idade dos participantes foi de 48,6 anos. Conforme a pontuação obtida com o questionário, os pacientes foram classificados segundo sua dependência nicotínica em cinco graus: muito baixa, baixa, média, elevada e muito elevada. Resultados: 54,9\% dos fumantes pertenciam ao

Grupo de Elevada Dependência Nicotínica ( $\geq 6$ pontos). Foi encontrada associação entre elevada dependência nicotínica e consumo diário de cigarros ou tempo até o fumar o primeiro cigarro do dia ( $<<0,001$ ). Conclusão: A utilização do Questionário de Tolerância de Fagerström mostrou ser de aplicação simples, rápida e de baixo custo, e permitiu identificar mais de $50 \%$ dos pacientes com um

grau de dependência nicotínica que faz prever desconforto ao deixar de fumar e necessidade de tratamento para controle da síndrome de abstinência. (J Pneumol 2002;28(4):180-186)
\end{abstract}

\section{Analysis of the use of the Fagerström Tolerance Questionnaire as an instrument to measure nicotine dependence}

Introduction: The University Hospital is reference in the city of Rio Grande, in the State of Rio Grande do Sul, Southeast part of Brazil, for chronic lung disease patients, with a major smoking cessation program. Aim: The purpose of this work was to analyze the utilization of Fagerström

Tolerance Questionnaire to assess the nicotine dependence of smoking patients with reference to individualization of treatment. Material and Method: The authors used the Fagerström Tolerance Questionnaire (FTQ) in daily smoking adult patients from the Medical Clinic and Chest Medicine Units of the University Hospital and Rio Grande Santa Casa Hospital, during the period of one year. Three hundred and one valid questionnaires were filled out, $40.5 \%$ by females and $59.5 \%$ by males. Mean age was 48.6 years. The score of the questionnaires classified patients by dependence into five degrees: very low, low, medium, high, and very high. Results: $54.9 \%$ belong to the group of high nicotine dependence (score $\geq 6$ ), with a significant correlation of elevated nicotine dependence and cigarettes per day or time to the first cigarette of the day ( $p<$

0.001). Conclusion: The use of the FTQ demonstrated ease of administration and more than $50 \%$ of the patients with a degree of chemical dependence that anticipate discomfort at cessation of smoking and need of treatment to control the withdrawal symptoms.

\footnotetext{
* Trabalho realizado no Departamento de Medicina Interna e Serviço de Pneumologia do Hospital Universitário da Fundação Universidade Federal do Rio Grande (FURG).

1. Professor Titular do Departamento de Medicina Interna.

2. Professora Adjunta do Departamento de Medicina Interna.

3. Residente do Serviço de Clínica Médica do Hospital Universitário.
}

4. Doutoranda do Curso de Medicina.

Endereço para correspondência - Dr. Luís Suárez Halty, Rua Canabarro, 235 - 96200-200 - Rio Grande, RS. Fax (53) 233-3970; email: lhalty@vetorialnet.com.br

Recebido para publicação em 8/3/02. Aprovado, após revisão, em 2/5/02. 
Descritores - Transtorno por uso de tabaco. Questionário. Síndrome de abstinência a substâncias. Tabagismo.

Key words - Tobacco use disorder. Questionnaires. Substance withdrawal syndrome. Smoking.

\section{INTRODUÇÃO}

Segundo dados do Centers for Disease Control, em $1995,24,7 \%$ da população adulta norte-americana $(\geq 15$ anos) era fumante regular e mais de dois terços (74\%) destes fumantes gostariam de parar de fumar ${ }^{(1)}$. No Rio Grande do Sul, em 1995, 27,4\% da população adulta era fumante regular e, destes, 69,2\% desejavam parar de fumar ${ }^{(2)}$. Em Rio Grande, RS, em 1994, entre adultos, achou-se prevalência de fumantes regulares de 23,8\%, dos quais $78 \%$ pretendiam parar de fumar ${ }^{(3)}$.

O Serviço de Doenças Respiratórias do Hospital Universitário é referência na cidade de Rio Grande para tratamento e acompanhamento de pneumopatas crônicos, sendo fundamental a existência de um programa que auxilie os pacientes a abandonarem o fumo, fator de risco dos mais importantes para pneumopatias e afecções cardiovasculares. Grande número de fumantes, apesar da intenção de abandonar o fumo, não consegue fazê-lo, o que pode justificar-se, em parte, por serem dependentes da nicotina. A nicotina é considerada uma droga psicotrópica ou psicoativa e a compreensão de sua função na determinação do tabagismo e na dependência do tabaco poderia ajudar o médico a tratar melhor seus pacientes fumantes.

O tabagismo representa um comportamento adquirido muito difundido e profundamente arraigado. Com exceção das necessidades primárias, é provável que o denominador comum mais próximo entre as pessoas seja o fumo. A esse respeito existem perguntas que continuam sendo um desafio, como:

1) Quais são os motivos pelos quais esse comportamento adquirido é reforçado?

2) Por que as pessoas continuam a fumar, apesar do conhecimento dos efeitos devastadores do fumo sobre a saúde?

3) Por que é tão difícil parar de fumar?

O papel crítico da dependência nicotínica e sua importância na persistência do fumo e na dificuldade para sua suspensão são bem conhecidos. Esta dependência é um processo complexo que envolve a inter-relação entre farmacologia (dependência física), componentes comportamentais (condicionamento) e/ou psicológico (dependência psicológica). É difícil avaliar, em cada caso, a real importância dos fatores condicionadores, da personalidade, das reações emocionais e das condições sociais.
Siglas e abreviaturas utilizadas neste trabalho QTF - Questionário de Tolerância de Fagerström GEDN - Grupo de elevada dependência nicotínica GBDN - Grupo de baixa dependência nicotínica $\mathrm{CO}$ - Monóxido de carbono TPC - Tempo até fumar o primeiro cigarro do dia CDC - Consumo diário de cigarros

À medida que se foram obtendo evidências, a opinião prevalente é de que a dependência nicotínica seria a chave da persistência do tabagismo e da dificuldade de sua suspensão e que, provavelmente, todos os fumantes regulares seriam dependentes da nicotina, ainda que em graus variáveis. Sendo assim, deve-se ter uma maneira de avaliar isso, já que os fumantes com elevada dependência, além da abordagem cognitivo-comportamental, necessitarão de terapia mais intensa, inclusive farmacológica, para lograr êxito no abandono do tabagismo. A fim de estimar o grau de dependência nicotínica é utilizado mundialmente, como ferramenta de avaliação, o Questionário de Tolerância de Fagerström ${ }^{(4)}$ em substituição a outros testes bem mais caros, que consomem mais tempo ou são invasivos.

Este estudo justifica-se pela expressiva prevalência de tabagismo encontrada na cidade de Rio Grande, assim como pelo elevado percentual de fumantes que desejam abandonar o tabagismo e não conseguem fazê-lo ou não conseguem ficar em abstinência(3). Assim, o presente trabalho tem como objetivo geral analisar a utilização do Questionário de Tolerância de Fagerström (QTF) como instrumento de medida da dependência nicotínica e, como objetivos específicos, identificar a magnitude da dependência nicotínica do tabagista atendido nos setores de Clínica Médica e Pneumologia do Hospital Universitário e Santa Casa e obter subsídios para planejar estratégias e organizar um programa abrangente e individualizado para cessação do tabagismo nessa população.

\section{CASUÍSTICA E MÉTODO}

O projeto foi executado no período de abril de 2000 a março de 2001, na cidade de Rio Grande, RS, nos fumantes regulares, adultos ( $\geq 15$ anos) atendidos nas áreas de internação de Clínica Médica do HU e Santa Casa e no setor de Pneumologia do HU. O método empregado foi a aplicação do instrumento de avaliação conhecido como Questionário de Tolerância de Fagerström (QTF), acrescido de dados de identificação, consumo de cigarros em maços/ano e motivação ou intenção de deixar o fumo nos próximos seis meses (Anexo 1). O questionário, uma vez obtida a aceitação do paciente para aplicação, foi preenchido pelo pneumologista assistente ou bolsista es- 
pecialmente treinado para esse fim. O contato foi aproveitado para motivar os doentes a entrarem no Programa de Cessação de Tabagismo do HU. O número de questionários válidos aplicados foi de 301 . Foi feita uma aplicação piloto a fim de avaliar a necessidade de realizar adaptações. Em forma aleatória, 10\% das entrevistas foram repetidas pelos pesquisadores como controle. Um dos problemas da utilização do Questionário de Fagerström é a determinação do ponto de corte entre fumantes com baixa e elevada dependência nicotínica. Tem sido utilizada a média ou mediana como ponto de corte ${ }^{(4)}$. Neste trabalho foi adotada a mediana.

As informações obtidas foram digitadas no banco de dados do Visual dBase versão 5.5, analisadas através do Epi-Info 6. Os resultados, depois de quantificados, foram avaliados pelo teste do qui-quadrado, admitindo-se uma possibilidade de erro alfa de $5 \%$.

\section{Resultados}

O questionário foi aplicado de forma válida a 301 fumantes regulares. A média de idade foi de 48,6 anos ( \pm 12,9), com idade mínima de 16 e máxima de 90 anos. Do total de entrevistados, 179 (59,5\%) eram do sexo masculino e $122(40,5 \%)$ do feminino. O consumo médio de cigarros em maços/ano foi de $42,4( \pm 32,1)$, com mediana de 35 e amplitude de um a 220 maços/ano. Quanto à motivação, 215 indivíduos $(71,5 \%)$ responderam estar motivados ou ter intenção de deixar o fumo nos próximos seis meses (Tabela 1 ).

\section{TABELA 1}

Perfil dos fumantes com relação a sexo, idade, consumo de cigarros em maços/ano e motivação para deixar o fumo

\begin{tabular}{|c|c|c|}
\hline Variáveis & $\mathbf{n}$ & $\%$ \\
\hline \multicolumn{3}{|l|}{ Sexo } \\
\hline - Masculino & 179 & 59,5 \\
\hline - Feminino & 122 & 40,5 \\
\hline \multicolumn{3}{|l|}{ Idade } \\
\hline - $\leq 30$ anos & 33 & 11,0 \\
\hline - $31-40$ & 48 & 16,0 \\
\hline - 41-50 & 90 & 29,8 \\
\hline - 51-60 & 69 & 22,9 \\
\hline - $\geq 61$ anos & 61 & 20,3 \\
\hline \multirow[t]{2}{*}{ Consumo de cigarros em maços/ano } & Mediana & Amplitude \\
\hline & 35 & 1 a 220 \\
\hline \multicolumn{3}{|l|}{ Motivação para deixar o fumo } \\
\hline - $\operatorname{Sim}$ & 215 & 71,5 \\
\hline • Não & 86 & 28,5 \\
\hline
\end{tabular}

Os resultados obtidos na aplicação de cada uma das seis perguntas do Questionário de Tolerância de Fagerström, assim como seus enunciados, estão mostrados na Tabela 2.

De acordo com a soma de pontos obtidos com as respostas referentes às seis perguntas da Tabela de Fagerström, classificou-se a dependência nicotínica em cinco graus: muito baixa, baixa, média, elevada e muito elevada (Tabela 3). O valor médio de dependência nicotínica no grupo estudado foi de 5,6 $( \pm 2,2)$ e a mediana de 6 sobre um total de 10 pontos. Dos 301 pacientes estudados, 165 (54,9\%) foram classificados no grupo de elevada ou muito elevada dependência.

O ponto de corte entre fumantes com baixa e elevada dependência nicotínica adotado neste trabalho foi a me-

TABELA 2

Perguntas do Questionário de Tolerância de Fagerström

Pergunta no 1: Quanto tempo após acor- Pontos n $\quad \%$ dar você fuma seu primeiro cigarro? $\quad 301 \quad 100$

- Dentro de 5 minutos

- Entre 6 e 30 minutos

- Entre 31 e 60 minutos

- Após 60 minutos

$3 \quad 119 \quad 39,5$

$2 \quad 110 \quad 36,5$

$\begin{array}{lll}1 & 38 & 12,7\end{array}$

Pergunta no 2: Você acha difícil não fumar em locais onde o fumo é proibido (como igrejas, bibliotecas, etc.)?

- Sim

- Não

15250,5

$149 \quad 49,5$

Pergunta no 3: Qual o cigarro do dia que traz mais satisfação (ou que mais detestaria deixar de fumar)?

- O primeiro da manhã

- Outros

$\begin{array}{lll}1 & 134 & 44,5 \\ 0 & 167 & 55,5\end{array}$

Pergunta no 4: Quantos cigarros você fuma por dia?

- 10 ou menos

- 11 a 20

- 21 a 30

- 31 ou mais

Pergunta no 5: Você fuma mais freqüentemente pela manhã (ou nas 1 as horas do dia) que no resto do dia?

- Sim

- Não

$1 \quad 78 \quad 25,9$

$\begin{array}{lll}0 & 223 & 74,1\end{array}$

Pergunta no 6: Você fuma mesmo quando está tão doente que precisa ficar de cama a maior parte do tempo?

- Sim

$211 \quad 70,1$

- Não

$0 \quad 90 \quad 29,9$


diana, cujo valor foi 6. Assim, os fumantes que somaram seis ou mais pontos (dependência elevada ou muito elevada) foram considerados como Grupo de elevada dependência nicotínica (GEDN: $54,9 \%$ ) e aqueles que somaram menos de seis pontos (dependência média, baixa ou muito baixa) foram catalogados como Grupo de baixa dependência nicotínica (GBDN: 45,1\%). A caracterização dos pacientes pertencentes ao grupo de elevada dependência nicotínica (GEDN) é o objetivo principal da aplicação do Questionário de Tolerância de Fagerström, já que trará conseqüências importantes no momento de decidir a conduta terapêutica.

Com relação ao grau de dependência nicotínica e gênero, não foi encontrada diferença entre homens e mulheres (Tabela 4).

Considerando faixa etária e dependência nicotínica, observou-se que o grupo de elevada dependência nicotínica foi aumentando com a idade até chegar ao máximo de $67,6 \%$ no grupo de 51 a 60 anos, para finalmente diminuir no grupo de $>60$ anos (Tabela 5).

As perguntas $\mathrm{n}^{\circ} 1$ e $\mathrm{n}^{\circ} 4$ do Questionário de Tolerância de Fagerström são as de maior peso, contribuindo cada uma com até três pontos do total de 10. A avaliação do tempo para fumar o primeiro cigarro após acordar (pergunta $\mathrm{n}^{\circ}$ 1) e dependência nicotínica mostrou que, quanto menor o tempo para fumar o primeiro cigarro, maior é o número de fumantes pertencentes ao grupo de elevada dependência nicotínica (GEDN). Do grupo de 165 pacientes com elevada dependência nicotínica (GEDN),

TABELA 3

Grau de dependência medido pela Tabela de Fagerström

\begin{tabular}{lcrr}
\hline Grau de dependência & Pontos & $\mathbf{n}$ & \% \\
\hline - Muito baixa & $0-2$ & 29 & 9,6 \\
- Baixa & $3-4$ & 50 & 16,6 \\
- Média & 5 & 57 & 18,9 \\
- Elevada & $6-7$ & 104 & 34,6 \\
- Muito elevada & $8-10$ & 61 & 20,3 \\
- Total & & 301 & 100 \\
\hline
\end{tabular}

TABELA 4

Gênero e grau de dependência nicotínica

\begin{tabular}{lrlrll}
\hline Gênero & \multicolumn{1}{c}{ GEDN } & GBDN & Total \\
\hline - Feminino & 70 & $57,4 \%$ & 52 & $42,6 \%$ & 122 \\
- Masculino & 95 & $53 \%$ & 84 & $46,9 \%$ & 179 \\
- Total & 165 & $54,8 \%$ & 136 & $45,2 \%$ & 301 \\
\hline
\end{tabular}

GEDN: grupo de elevada dependência nicotínica ( $\geq 6$ pontos)

GBDN: grupo de baixa dependência nicotínica

$p>0,5$

J Pneumol 28(4) - jul-ago de 2002
111 , ou sejam, $67,3 \%$ deles, fumam seu primeiro cigarro nos cinco minutos logo após acordar, enquanto somente oito (5,9\%) dos 136 do grupo de baixa dependência nicotínica (GBDN) o fazem nesse tempo. Quando consideramos, especificamente, o grupo de 119 fumantes que fumam nos primeiros cinco minutos, tem-se que destes, 111 $(93,3 \%)$ fazem parte do GEDN (Tabela 6 e Figura 1).

TABELA 5

Idade e grau de dependência nicotínica

\begin{tabular}{lrlrlrl}
\hline \multicolumn{1}{c}{ Idade } & \multicolumn{2}{c}{ GEDN } & \multicolumn{2}{c}{ GBDN } & \multicolumn{2}{c}{ Total } \\
\hline - $\leq 30$ anos & 12 & $36,3 \%$ & 21 & $6,4 \%$ & 33 & $11 \%$ \\
- $31-40$ anos & 25 & $52,1 \%$ & 23 & $47,9 \%$ & 48 & $15,9 \%$ \\
- $41-50$ anos & 54 & $60 \%$ & 36 & $40 \%$ & 90 & $29,9 \%$ \\
- $51-60$ anos & 46 & $67,7 \%$ & 23 & $33,3 \%$ & 69 & $22,9 \%$ \\
- $\geq 61$ anos & 28 & $45,9 \%$ & 33 & $54,1 \%$ & 61 & $20,3 \%$ \\
- Total & 165 & $54,8 \%$ & 136 & $45,2 \%$ & 301 & $100 \%$ \\
\hline
\end{tabular}

GEDN: grupo de elevada dependência nicotínica

GBDN: grupo de baixa dependência nicotínica $\chi^{2}: 11,53 ; 4 \mathrm{GL} ; \mathrm{p}=0,02$

TABELA 6

Tempo para fumar o primeiro cigarro e grau de dependência nicotínica

\begin{tabular}{lccrrr}
\hline $\begin{array}{l}\text { Tempo para fumar } \\
\text { o primeiro cigarro }\end{array}$ & $\begin{array}{c}\text { Total } \\
\mathbf{3 0 1}\end{array}$ & $\begin{array}{c}\text { GEDN } \\
\mathbf{1 6 5}\end{array}$ & \multicolumn{2}{c}{$\begin{array}{c}\text { GBDN } \\
\mathbf{1 3 6}\end{array}$} \\
\hline - $<5$ min & 119 & $111 / 119$ & $93,3 \%$ & $8 / 119$ & $6,7 \%$ \\
- > 5 a 30min & 110 & $45 / 110$ & $40,9 \%$ & $65 / 110$ & $59,1 \%$ \\
- > 30 a 60min & 38 & $6 / 38$ & $15,8 \%$ & $32 / 38$ & $84,2 \%$ \\
- > 60min & 36 & $3 / 34$ & $8,8 \%$ & $31 / 34$ & $91,2 \%$ \\
\hline
\end{tabular}

GEDN: grupo de elevada dependência nicotínica ( 26 pontos)

GBDN: grupo de baixa dependência nicotínica

$\chi^{2}=13,2 ; 3 \mathrm{GL} ; \mathrm{p}<0,001$

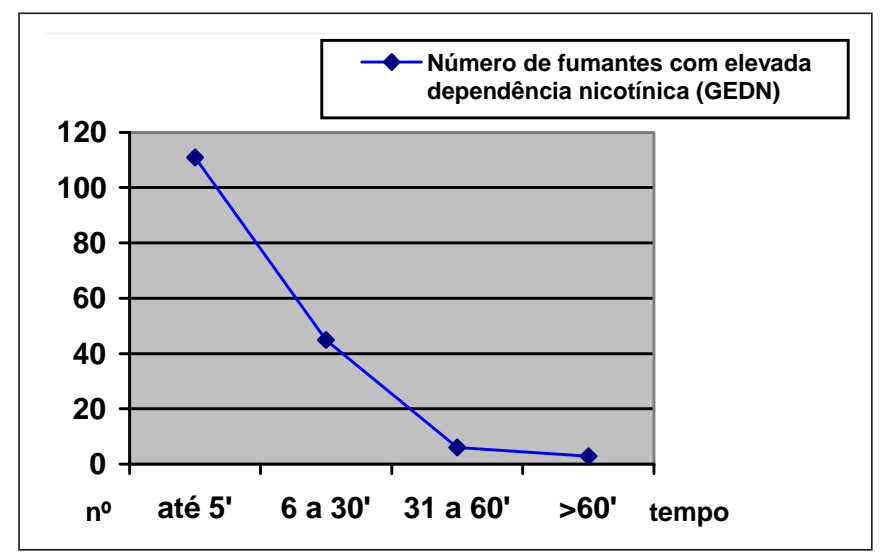

Figura 1 - Tempo em minutos para fumar o primeiro cigarro e número de fumantes com elevada dependência nicotínica 
A apreciação do número de cigarros fumados por dia (pergunta $\mathrm{n}^{\mathrm{O}} 4$ ) e grau de dependência nicotínica mostrou que, quanto maior o número de cigarros fumados, maior é o número de fumantes pertencentes ao grupo de elevada dependência nicotínica (GEDN) com $p<0,001$. Dos 155 pacientes que fumam $\geq 21$ cigarros por dia, 128 $(82,5 \%)$ pertencem ao GEDN (Tabela 7 e Figura 2).

Não foi encontrada associação entre a intenção ou motivação dos fumantes para abandonar o fumo nos próximos seis meses e seu grau de dependência nicotínica (Tabela 8).

TABELA 7

Consumo diário de cigarros e grau de dependência nicotínica

\begin{tabular}{ccrrrr}
\hline $\begin{array}{c}\text { No de cigarros } \\
\text { fumados por dia }\end{array}$ & $\begin{array}{c}\text { Total } \\
\mathbf{3 0 1}\end{array}$ & \multicolumn{2}{c}{$\begin{array}{c}\text { GEDN } \\
\mathbf{1 6 5}\end{array}$} & \multicolumn{2}{c}{$\begin{array}{c}\text { GBDN } \\
\mathbf{1 3 6}\end{array}$} \\
\hline - $\leq 10$ & 27 & 2 & $7,4 \%$ & 25 & $92,6 \%$ \\
- 11 a 20 & 119 & 35 & $29,4 \%$ & 84 & $70,6 \%$ \\
- 21 a 30 & 84 & 64 & $76,2 \%$ & 20 & $23,8 \%$ \\
- > 30 & 71 & 64 & $90,1 \%$ & 7 & $9,9 \%$ \\
\hline
\end{tabular}

GEDN: grupo de elevada dependência nicotínica ( $\geq 6$ pontos)

GBDN: grupo de baixa dependência nicotínica

$\chi^{2}=10,67 ; 3 \mathrm{GL} ; \mathrm{p}<0,001$

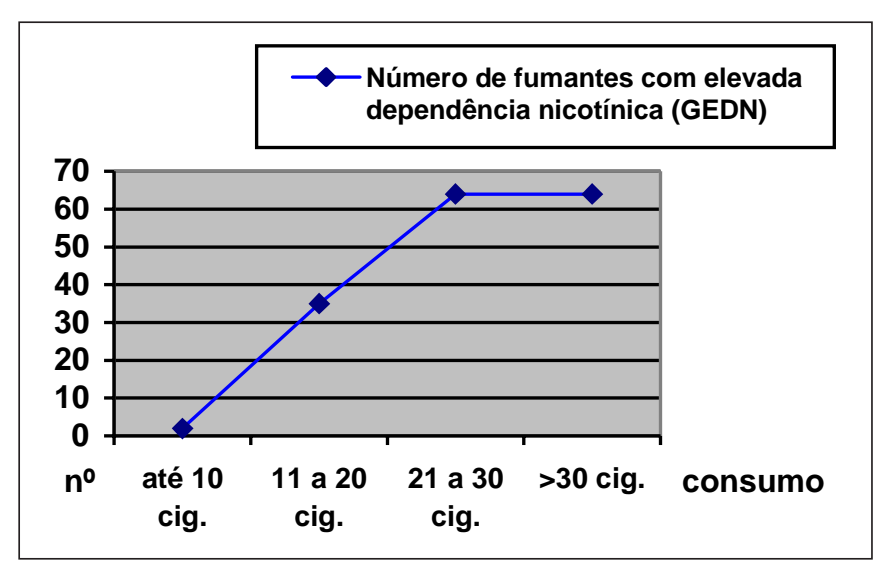

Figura 2 - Consumo diário de cigarros e número de fumantes com elevada dependência nicotínica

TABELA 8

Motivação para abandonar o fumo e grau de dependência nicotínica

\begin{tabular}{lrrrr}
\hline & \multicolumn{2}{c}{ GEDN } & \multicolumn{2}{c}{ GBDN } \\
& $\mathbf{1 9 6}$ & $\mathbf{7 1 , 5 \%}$ & $\mathbf{7 8}$ & $\mathbf{2 8 , 5} \%$ \\
\hline Motivados a abandonar o fumo & 107 & $54,6 \%$ & 44 & $56,4 \%$ \\
Não motivados a abandonar o fumo & 89 & $45,4 \%$ & 34 & $43,6 \%$ \\
\hline
\end{tabular}

\section{DISCUSSÃO}

A nicotina é considerada uma droga psicoativa que exerce atividade estimulante do sistema nervoso central(5). Dependência nicotínica significa usar compulsivamente o fumo, ser incapaz de parar de fumar ou de manter-se sem fumar depois de parar, ou ter dificuldade de tentar parar por aparição de síndrome de abstinência e/ou desejo imperioso de fumar ("fissura"). Tanto a dependência nicotínica como sua síndrome de abstinência estão classificadas como sendo doenças com critérios bem definidos ${ }^{(6)}$.

O Questionário de Tolerância de Fagerström (QTF) originariamente constava de oito perguntas curtas, que posteriormente foram reduzidas para as seis atuais, com revisão e ampliação das categorias de contagem nas perguntas 1 e 4 , sugerindo modificar sua denominação para Questionário de Dependência Nicotínica de Fagerström ${ }^{(4)}$. Ele fundamenta-se em seis suposições maiores hipotéticas com as quais estaria relacionada a dependência nicotínica e que seriam o reflexo fiel do comportamento frente ao fumo, independentemente das interpretações pessoais $^{(4,7-9)}$

Da aplicação do Questionário de Tolerância de Fagerström pode-se salientar especificamente que:

A pergunta $n^{\circ} 1$, que se refere ao tempo de fumar 0 primeiro cigarro após acordar, é uma das duas perguntas mais importantes e avalia quão rápido o fumante precisa de um cigarro pela manhã, revelando a intensidade da "fissura" (episódios transitórios de desejo imperioso de fumar). A nicotina tem vida média relativamente curta. Os fumantes dependentes terão, ao acordar, baixo nível sérico dela e experimentarão sintomas de abstinência se não fumarem rapidamente seu primeiro cigarro do dia. Ou seja, essa pergunta, teoricamente, seria um preditor poderoso da dependência nicotínica. Na amostra apresentada, o máximo poder discriminatório dessa pergunta foi alcançado com o tempo $\leq 5$ minutos. Dos 119 pacientes desse grupo, 93,3\% pertencem ao de elevada dependência nicotínica (GEDN) e somente 6,7\% ao de baixa dependência nicotínica (GBDN). Quando se usou o intervalo de tempo até 30 minutos, como recomendado por alguns autores, caiu o poder discriminatório, sendo que dos 229 pacientes, 68,2\% pertenciam ao GEDN e 31,8\% ao GBDN.

A avaliação da pergunta $n^{\circ} 2$, que se refere ao comportamento do fumante nos lugares onde o fumo é proibido, é complicada, já que cada vez existem mais leis que próibem o fumo em diferentes locais. Muitos fumantes fumam intensamente antes de entrar (carregam-se de nicotina) e, como conseqüência, expressam não ter dificuldade em não fumar em locais de proibição. Esta pergunta e a $\mathrm{n}^{\circ} 3$, que se refere ao cigarro que traz maior satisfação, necessitam de certa introspecção para ser respon- 
didas e poderiam ser mais importantes como indicadores comportamentais.

Quanto à pergunta $\mathrm{n}^{\mathrm{0}} 4$, sobre o consumo diário de cigarros, trata-se da segunda das duas perguntas mais importantes do QTF e mede a quantidade de nicotina à qual o indivíduo se tornou dependente. Nesta amostra encontraram-se 155 (51\%) fumantes de $\geq 21$ cigarros por dia, sendo que destes, $82,6 \%$ pertencem ao GEDN $e$ somente $17,4 \%$ ao GBDN.

A pergunta $n^{\circ} 5$, que trata de fumar mais pela manhã do que no restante do dia, é uma das três que avaliam o assunto fumo matinal. Neste caso, só $25,9 \%$ responderam afirmativamente, sendo, portanto, uma pergunta com pouco poder discriminatório.

A pergunta $n^{\circ} 6$, que se refere ao fumar ainda que doente, faria parte da apreciação do assunto consumo de cigarros. Na amostra em análise, 70,1\% dos entrevistados atestaram que sim, indicando ser esta pergunta, também, um preditor importante de dependência nicotínica.

Analisando o total da amostra observou-se que:

1) $54,9 \%$ pertencem ao grupo de elevada dependência nicotínica ( $\geq 6$ pontos) e, provavelmente, estes pacientes terão desconforto (síndrome de abstinência) ao deixar de fumar(5).

2) Não existe diferença de grau de dependência elevado/muito elevado (GEDN) quanto à distribuição por gênero $(p>0,5)$.

3) A proporção dos fumantes pertencentes ao GEDN aumenta progressivamente nos grupos etários, até chegar ao máximo de $67,6 \%$ no grupo de 51 a 60 anos.

4) Existe associação estatisticamente significante ( $p<$ $0,001)$ entre o grupo de elevada dependência nicotínica (GEDN) e tempo para fumar o primeiro cigarro do dia.

5) Existe associação estatisticamente significante ( $p<$ 0,001 ) entre o grupo de elevada dependência nicotínica (GEDN) e consumo diário de cigarros.

6) Relacionando motivação e dependência, existem os não motivados e com elevada dependência (GEDN), que seriam o pior grupo para o abandono do fumo e representam $14,7 \%$ do total, e os motivados e com baixa dependência (GBDN), que seriam o melhor grupo e representam 29,7\% do total. Porém, não foi encontrada associação entre dependência e motivação para abandonar o fumo $(p>0,5)$.

O objetivo do Questionário de Tolerância de Fagerström é a identificação e a medida da dependência nicotínica com finalidade de aproveitar o resultado como um elemento de ajuda na decisão do tratamento do tabagismo. Quanto mais dependente da nicotina for o fumante, maior será a possibilidade e/ou a gravidade da síndrome de abstinência durante a suspensão do tabagismo, assim como maior será a dificuldade de manter a abstinência em longo prazo.

Segundo Heatherton et al. ${ }^{(4)}$, O QTF revisado tem um aceitável nível de consistência interna e sua pontuação final correlaciona-se significativamente com os níveis dos marcadores bioquímicos de elevada sensibilidade e especificidade para tabagismo e dependência nicotínica, como são os níveis de monóxido de carbono no ar expirado $e$ os níveis séricos, urinários ou na saliva de nicotina (sensíveis ao fumo nas últimas horas) e de cotinina (sensível ao fumo nos últimos sete dias) ${ }^{(4,8)}$. Ainda, deve-se ter presente que os níveis de nicotina podem não ser equivalentes ao grau de dependência e que alguns indivíduos podem satisfazer-se com níveis relativamente baixos e ainda assim ser dependentes. O nível individual de resposta à nicotina pode ser diferente, talvez por razões genéticas.

O grau de associação entre o número de pontos obtidos na aplicação do questionário e a síndrome de abstinência é mais fraco, assim como é baixo também o valor preditivo dos níveis de monóxido de carbono (CO), nicotina, cotinina e aparição desta síndrome ${ }^{(8)}$. Essa síndrome não estaria só ligada à privação de nicotina. Existiriam diferenças individuais já apontadas antes. Inclusive, alguns dos sintomas da síndrome podem estar relacionados à perda de rituais comportamentais e/ou de reforços sensoriais. Porém, continua claro que a chave do aparecimento da síndrome de abstinência é a dependência à nicotina.

Examinando criticamente a amostra e com o intuito de simplificar, poderia decidir-se pelo apoio à posição de somente utilizar as perguntas no 1 e $\mathrm{n}^{\circ} 4$ do QTF, que tratam do tempo até fumar o primeiro cigarro do dia (TPC) e do consumo diário de cigarros $(\mathrm{CDC})$, já que são quantitativamente as de maior peso e tem correlação estatisticamente significante $(\mathrm{p}<0,001)$ com o grupo de elevada dependência nicotínica (GEDN), especialmente quando se utilizam como divisórias TPC $\leq 5$ minutos e CDC de $\geq 21$ cigarros. Essas perguntas já foram utilizadas por Heatherton et al. com a designação de Índice de Intensidade do Tabagismo ${ }^{(4)}$ e estão incluídas entre as seis perguntas denominadas Perguntar e Avaliar no Consenso do MSINCA $^{(10)}$. Porém, deve concordar-se com a posição de que o QTF precisa ser utilizado na íntegra e ser analisado como um instrumento de avaliação de dependência nicotínica que se fundamenta na apreciação equilibrada de dois fatores principais $^{(4)}$ : 1 - Fumo matinal através de (em ordem decrescente de importância): a) Tempo até fumar o primeiro cigarro do dia (TPC); b) Fumar mais pela manhã; c) Detestar deixar o primeiro cigarro da manhã. 2 - Consumo de cigarros através de (em ordem decrescente de importância): a) Consumo diário de cigarros (CDC); b) Fumar ainda doente; c) Fumar em lugares proibidos. 
Deixar de fumar é um processo complexo, não é simples nem fácil e requer uma abordagem cognitivo-comportamental associada ou não a farmacoterapia específica. Nesse processo todos os recursos para avaliar as necessidades individuais, o grau de dependência nicotínica e a disponibilidade de parar o fumo devem ser utilizados.

Concluindo, a utilização do (QTF) mostrou-se de aplicação simples, rápida e de baixo custo. Sendo o QTF um instrumento confiável de avaliação do grau de dependência nicotínica, sua utilização deve ser rotineira na anamnese de todo doente tabagista. O QTF deve ser utilizado na íntegra e seria aconselhável associá-lo à utilização dos estágios de mudança de Prochaska e DiClemente, já que estes apontam para a análise dos aspectos emocionais do hábito tabágico(11). A identificação dos pacientes pertencentes ao grupo de elevada dependência nicotínica GEDN ( $\geq 6$ pontos) - é o objetivo principal da aplicação do QTF. O resultado de $54,9 \%$ dos fumantes pertencentes ao GEDN faz prever a possibilidade de ocorrência e/ou gravidade da síndrome de abstinência, a necessidade do tratamento de substituição e maior dificuldade de manter a abstinência em longo prazo, no caso de estes entrarem num programa de cessação do fumo. Não foi encontrada associação entre dependência nicotínica e motivação para parar de fumar $(p>0,5)$.

\section{REFERÊNCIAS}

1. Centers for Disease Control: Cigarette smoking among adults. USA, 1995. MMVR 1997;46:1217-20.

2. Oliveira Netto IC. Epidemiologia do tabagismo no Rio Grande do Sul [tese]. Rio Grande do Sul, Universidade Federal do Rio Grande do Sul; 1998.

3. Halty L, Hüttner MD, Santos A, Coelho C, Gruber R. Tabagismo em Rio Grande, RS (resumo). J Pneum 1994;20:118-391.

4. Heatherton TF, Kozlowski LT, Frecker RC, Fagerström KO. The Fagerström test for nicotine dependence: a revision of the Fagerström Tolerance Questionnaire. Br J Addict 1991;86:1119-27.

5. Brasil, Ministério da Saúde. Instituto Nacional do Câncer: Ajudando seu paciente a deixar de fumar. INCA, 1997; 21.

6. American Psychiatric Association. Diagnostic and statistical manual of mental disorders. $4^{\text {th }}$ ed. Washington, 1994.

7. Fagerström KO. Measuring degree of physical dependence to tobacco smoking with reference to individualization of treatment. Addict Behav 1978;3:235-41.

8. Fagerström KO, Schneider NG. Measuring nicotine dependence: a review of the Fagerström Tolerance Questionnaire. J Behav Med 1989; 12:159-82.

9. Heatherton TF, Kozlowski LT. Nicotine addiction and its assessment. Ear Nose Throat J 1992;69:763-7.

10. Brasil, Ministério da Saúde, Instituto Nacional do Câncer-INCA-CONPREV: Abordagem e tratamento do fumante. Consenso 2001, Rio de Janeiro, INCA, 2001.

11. Prochaska JO, DiClemente CC. Stages and processes of self-change of smoking: toward an integrative model of change. J Consult Clin Psychol 1983;51:390.

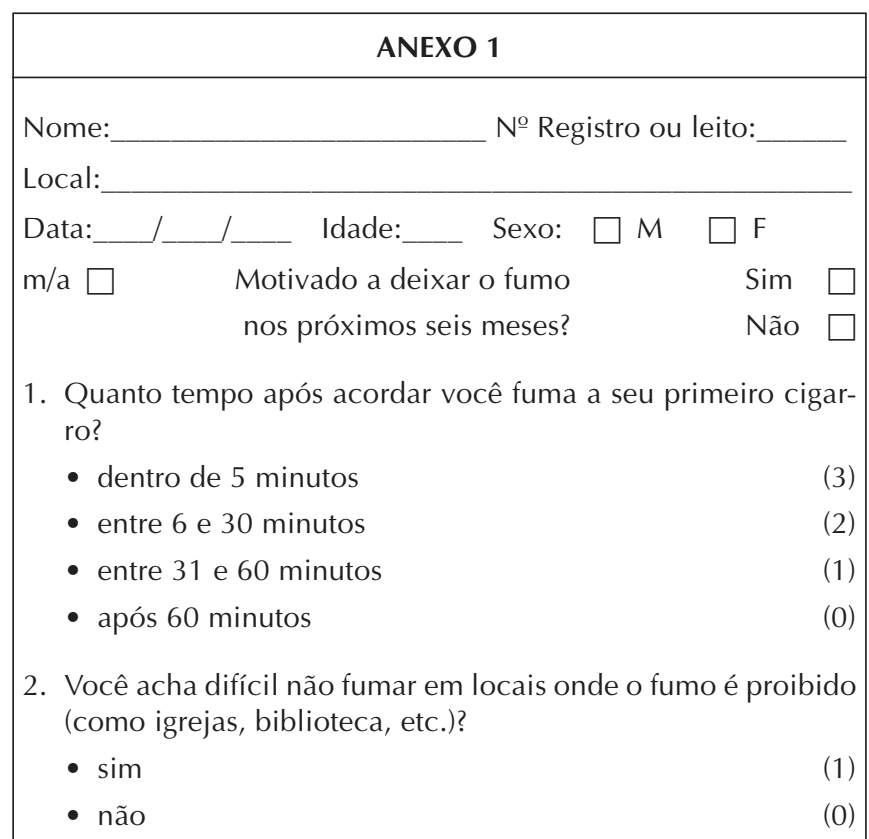

3. Qual o cigarro do dia que traz mais satisfação (ou que mais detestaria deixar de fumar)?

- o primeiro da manhã

- outros

4. Quantos cigarros você fuma por dia?

- 10 ou menos

(0)

- 11 a 20

- 21 a 30

- 31 ou mais

5. Você fuma mais freqüentemente pela manhã (ou nas primeiras horas do dia) que no resto do dia?

- Sim

- Não

6. Você fuma mesmo quando está tão doente que precisa ficar de cama a maior parte do tempo?

- Sim

- Não

Total:

\section{Tabela de Fagerström}

Conclusão quanto ao grau de dependência

- 0 a 2 pontos - muito baixo

- 3 a 4 pontos - baixo

- 5 pontos - médio

- 6 a 7 pontos - elevado

- 8 a 10 pontos - muito elevado

Uma soma acima de seis pontos indica que provavelmente o paciente terá desconforto (síndrome de abstinência) ao deixar de fumar ${ }^{(5)}$. 